\title{
Coalitional Games in Gaussian Interference Channels
}

\author{
Suhas Mathur, Lalitha Sankaranarayanan and Narayan B. Mandayam \\ WINLAB Dept. of Electrical and Computer Engineering \\ Rutgers University, Piscataway, NJ \\ \{suhas, lalitha, narayan\}@winlab.rutgers.edu
}

\begin{abstract}
The formation of coalitions in a Gaussian interference channel comprised of $M$ transmit-receive links where the receivers are allowed to cooperate is studied under the framework of coalitional game theory. Allowing any arbitrary sharing of the total rate achieved by a coalition between its member links, it is shown that the grand coalition (coalition of all links) maximizes spectrum utilization and is also stable, that is, the links in this coalition have no incentives to leave and form other coalitions. The issue of fairness in allocating rates to members of a grand coalition is addressed via a Nash bargaining solution where each link utility is modeled as the rate gained by being in a coalition relative to the rate achieved in the interference channel. Further, a rate allocation solution using proportional fairness is also presented and the results are illustrated with examples.
\end{abstract}

\section{INTRODUCTION}

We consider a Gaussian interference channel [1] comprised of a number of communication links where a link corresponds to a single transmitter-receiver pair as shown in Fig. 1. Except for certain special cases (see [1]), the capacity region of this channel remains an open problem. Recently, cooperation between transmitters and/or receivers in communication networks has been shown to mitigate interference between radio links and lead to rate and diversity gains (see for e.g. [2], [3] and the references therein).

We analyze the effect of cooperation between the receivers of a $M$-link interference channel under the framework of coalitional game theory. When a set of links cooperate to form a coalition, we assume that the receivers of these links jointly decode their received signals. Further, we assume that the signals from the links that are not part of this coalition are treated as interference by the receivers in the coalition. The value of a coalition is the maximum information-theoretic rate that can be achieved between the transmitters and the receivers in the coalition. Depending on the share of the value that a link receives while in a coalition, it can choose to leave or remain in the coalition. The formation of coalitions is studied in [4] for the Gaussian multi-access channel where the interfering transmitters bargain for rates by threatening to transmit worst case jamming noise. For the interference channel considered here, we determine the coalitions formed when the receivers of mutually interfering radios cooperate and each link within a coalition can be assigned an arbitrary share of the total value. Under these conditions, we show that the grand coalition

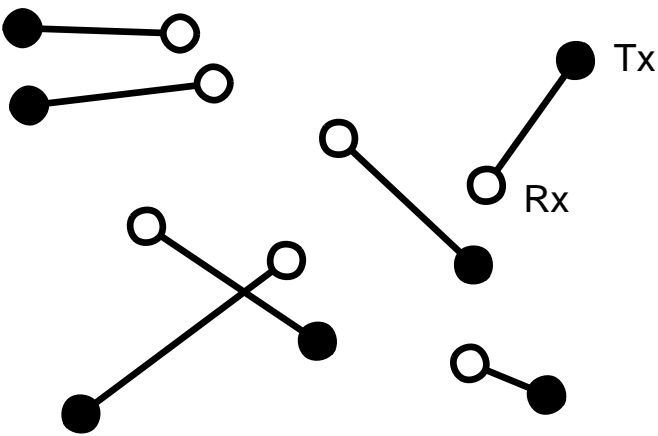

Fig. 1. An interference channel with $M$ transmit-receive links

maximizes spectrum utilization and is also stable. We further propose a Nash bargaining solution and a proportional fair solution as two approaches to allocate rate to the members of a grand coalition; specifically the Nash solution allocates rates by maximizing the product of the rate gains that each link achieves via receiver cooperation relative to that achieved in the interference channel.

\section{SySTEM MODEL}

We consider an interference channel of $M$ communication links, each formed by a single transmitter-receiver pair, coexisting in the same shared spectrum [1]. $\mathcal{X}_{m}$ is the input alphabet of the transmitter of link $m$ and $\mathcal{Y}_{m}$ is the output alphabet at the corresponding receiver. We denote by $\mathcal{S}=$ $\{1,2, \ldots, M\}$ the set of all links and write $X_{\mathcal{G}}=\left\{X_{m}: m \in\right.$ $\mathcal{G}\}$ for all $\mathcal{G} \subseteq \mathcal{S}$ and $\mathcal{G}^{c}$ as the complement of $\mathcal{G}$ in $\mathcal{S}$. We consider an additive white Gaussian noise channel with flat fading. The channel is used $n$ times. The received signal at the receiver of link $m$ in the $i^{t h}$ time instant is given by

$$
Y_{m, i}=\sum_{k=1}^{M} h_{m, k, i} X_{k, i}+Z_{m, i}
$$

where $X_{m, i} \in \mathcal{X}_{m}, Y_{m, i} \in \mathcal{Y}_{m}$, and $h_{m, k}$ is the channel gain between the transmitter of link $k$ to the receiver of link $m$ and is assumed known at the receiver. We also assume the channel gains remain unchanged over the $n$ channel uses. The noise entries $Z_{m, i} \sim \mathcal{C N}(0,1)$, are independent, circularly 
symmetric, complex Gaussian random variables with zero mean and unit variance, for all $m$. The power constraint at the $m^{\text {th }}$ transmitter is

$$
\sum_{i=1}^{n} E\left|X_{m, i}\right|^{2} \leq n P_{m} \quad m \in \mathcal{S}
$$

The capacity region of the interference channel that results when links do not cooperate is, in general, unknown. For the interference channel considered here, we assume that the transmitters employ Gaussian signaling subject to a power constraint (2). We also assume that receivers that choose to cooperate communicate with one another via a side channel or through a central agent such as a spectrum server [5], [6]. Further, we assume that the transmitters do not cooperate. This models a variety of practical networks operating in the unlicensed bands where the receivers can communicate via a backbone network while the wireless transmitters, in general, cannot. For the input signaling considered, a coalition of cooperating receivers treats signals from transmitters outside the coalition as additive white Gaussian noise. Such a coalition can be modeled as a single-input, multiple-output multiple access channel (SIMO-MAC), the capacity region of which is known [7] and is achieved by the Gaussian input signaling chosen.

We use a value $v(\mathcal{G})$ of a coalition of links $\mathcal{G}$ (precise definitions are given in Section III) to denote the maximum sum-rate achievable by the links in $\mathcal{G}$. For the channel model considered, $v(\mathcal{G})$ is then the mutual information between the transmitters and receivers in $\mathcal{G}$ given as

$$
v(\mathcal{G})=\max _{\underline{R}_{\mathcal{G}} \in \mathcal{C}_{\mathcal{G}}} \sum_{i \in \mathcal{G}} R_{i}=I\left(X_{\mathcal{G}} ; Y_{\mathcal{G}}\right)
$$

where $\underline{R}_{\mathcal{G}}=\left(R_{m}\right)_{m \in \mathcal{G}}$ is the vector of rates for links in $\mathcal{G}$ and $\mathcal{C}_{\mathcal{G}}$ is the capacity region of the SIMO-MAC formed by the users in $\mathcal{G}$. The value $v(\mathcal{G})$ of a coalition $\mathcal{G}$ can be apportioned between its members in any arbitrary manner. Depending on its allocated share of $v(\mathcal{G})$, a receiver may decide to break away from the coalition $\mathcal{G}$ and join another coalition where it achieves a greater rate. We model the problem of determining the stable coalitions and the resulting rate allocations for the interference channel as a coalitional game and refer to this game as the interference channel coalitional game. In the following section, we use results from coalitional game theory to prove the existence of stable rate-maximizing coalitions for the network considered.

\section{Coalitional Games in Receiver Cooperation NETWORKS}

We first briefly review coalitional game theory [8] and present definitions in the context of our problem. We consider a coalitional game in which every coalition of links is ascribed a single number, interpreted as the payoff available to the coalition. The share of payoff received by the links in a coalition is called a payoff vector. When there are no restrictions on how this payoff may be apportioned among the members of the coalition, the game is said to have transferable payoff.

Definition 1: A coalitional game with transferable payoff $\langle\mathcal{S}, v\rangle$ consists of

- a finite set of links $\mathcal{S}$,

- a function $v$ that associate with every non-empty subset $\mathcal{G}$ (a coalition) of $\mathcal{S}$, a real number $v(\mathcal{G})$ (the value of $\mathcal{G}$ ).

Receiver cooperation through joint decoding results in coalitions where the value of each coalition is not influenced by the actions of players outside that coalition. This is because the maximum sum-rate $v(\mathcal{G})$ achievable by such a coalition $\mathcal{G}$ depends on the players in $\mathcal{G}^{c}$ only through the interference offered by them, which in turn is independent of coalitions formed within $\mathcal{G}^{c}$. In general, however, $v(\mathcal{G})$ may depend upon the actions of links outside the coalition $\mathcal{G}$. For example, if transmitters were allowed to cooperate in our model it would lead to such a situation.

Definition 2: A coalitional game with transferable payoff is said to be superadditive if for any two disjoint coalitions $\mathcal{G}_{1}, \mathcal{G}_{2} \subseteq S, v\left(\mathcal{G}_{1} \cup \mathcal{G}_{2}\right) \geq v\left(\mathcal{G}_{1}\right)+v\left(\mathcal{G}_{2}\right)$.

Theorem 3: The grand coalition maximizes spectrum utilization in the interference channel coalitional game.

Proof: From definition 2, for a superadditive game, the sum-rate of all links is maximized by the coalition formed by all links, namely the grand coalition. For the interference channel coalitional game, maximizing the sumrate is equivalent to maximizing the utilization of the shared spectrum. Therefore, we only need to show that the value of a coalition $v(\mathcal{G})$ for the interference channel coalitional game, defined in (3), is a superadditive function.

Consider two coalitions $\mathcal{G}_{1}$ and $\mathcal{G}_{2}$ such that $\mathcal{G}_{1} \cap \mathcal{G}_{2}=\phi$. In order to prove that $v(\mathcal{G})$ is superadditive, we need to show that

$$
I\left(X_{\mathcal{G}_{1} \cup \mathcal{G}_{2}} ; Y_{\mathcal{G}_{1} \cup \mathcal{G}_{2}}\right) \geq I\left(X_{\mathcal{G}_{1}} ; Y_{\mathcal{G}_{1}}\right)+I\left(X_{\mathcal{G}_{2}} ; Y_{\mathcal{G}_{2}}\right)
$$

We expand $I\left(X_{\mathcal{G}_{1} \cup \mathcal{G}_{2}} ; Y_{\mathcal{G}_{1} \cup \mathcal{G}_{2}}\right)$ as

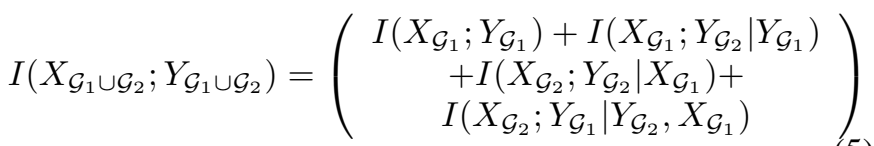

Comparing (5) with (4), since mutual information is nonnegative, to prove (4), we only need to show that the third term in the expansion above is greater than $I\left(X_{\mathcal{G}_{2}} ; Y_{\mathcal{G}_{2}}\right)$. Expanding $I\left(X_{\mathcal{G}_{2}} ; Y_{\mathcal{G}_{2}} \mid X_{\mathcal{G}_{1}}\right)$, we have

$$
\begin{aligned}
I\left(X_{\mathcal{G}_{2}} ; Y_{\mathcal{G}_{2}} \mid X_{\mathcal{G}_{1}}\right) & =H\left(X_{\mathcal{G}_{2}}\right)-H\left(X_{\mathcal{G}_{2}} \mid Y_{\mathcal{G}_{2}}, X_{\mathcal{G}_{1}}\right) \\
& \geq H\left(X_{\mathcal{G}_{2}}\right)-H\left(X_{\mathcal{G}_{2}} \mid Y_{\mathcal{G}_{2}}\right) \\
& =I\left(X_{\mathcal{G}_{2}} ; Y_{\mathcal{G}_{2}}\right)
\end{aligned}
$$

where we have exploited the independence of the transmitter signals in (6) and that conditioning reduces entropy to obtain the inequality in (7). Thus, the interference channel coalitional game is a superadditive game. 
We remark that, without superadditivity, finding the optimal coalition structure (partition of links into coalitions) is an $\mathcal{N P}$-complete problem [9]. This is because the number of possible coalition structures, given by the Bell number, grows exponentially fast with $M$.

Definition 4: For any coalition $\mathcal{G}$, a vector $\left(x_{m}\right)_{m \in \mathcal{G}}$ of real numbers is a $\mathcal{G}$-feasible payoff vector if $x(\mathcal{G})=\sum_{m \in \mathcal{G}} x_{m}=$ $v(\mathcal{G})$. The $\mathcal{S}$-feasible payoff vector is referred to as a feasible payoff profile.

Of all possible coalitions that can form, the coalitions that are stable, that is, those whose member links have no incentives to leave, are of most interest. The set of such stable coalitions comprises a core, defined formally below.

Definition 5: The core, $C(v)$, of a coalitional game with transferable payoff, $\langle\mathcal{S}, v\rangle$, is the set of feasible payoff profiles $\left(x_{m}\right)_{m \in \mathcal{S}}$ for which there is no coalition $\mathcal{G} \subset \mathcal{S}$ and a corresponding $\mathcal{G}$-feasible payoff vector $\left(y_{m}\right)_{m \in \mathcal{G}}$ such that $y_{m}>x_{m}$ for all $m \in \mathcal{G}$.

From definition 5, it follows that the set of feasible payoff profiles in the core satisfy $v(\mathcal{G}) \leq x(\mathcal{G})$ for every coalition $\mathcal{G} \subseteq \mathcal{S}$. We claim that this condition is equivalent to definition 5 . This is because in a game with transferable payoff if there exists a coalition $\mathcal{G}$ with $v(\mathcal{G})>x(\mathcal{G})$ then we can always find a $\mathcal{G}$-feasible payoff vector $\left(y_{m}\right)_{m \in \mathcal{G}}$ such that $y_{m}>x_{m}$, for all $m \in \mathcal{G}$. Such an assignment can result, for instance, when the $\mathcal{G}$-feasible payoff vector $\left(y_{m}\right)_{m \in \mathcal{G}}$ is constructed by assigning to each link $m \in \mathcal{G}$, the payoff $x_{m}$ and then uniformly apportioning the surplus payoff $v(\mathcal{G})-x(\mathcal{G})$ between links in $\mathcal{G}$. We use this equivalent definition of the core in the following theorem.

In general, the core of a coalitional game can be empty. We now prove that the core of the interference channel coalitional game is non-empty.

Theorem 6: The interference channel coalitional game with receiver cooperation and transferable utility has a non-empty core.

Proof: Since the interference channel coalitional game is superadditive, we need only consider the definition of the core in the context of the grand coalition. Consider a feasible payoff profile $\underline{R}_{\mathcal{S}}=\left(R_{m}\right)_{m \in \mathcal{S}}$ that lies in the capacity region of the SIMO-MAC $\mathcal{C}_{\mathcal{S}} \cdot \mathcal{C}_{\mathcal{S}}$ is the capacity region that results when all links cooperate to form a grand coalition resulting in $M$ independent transmitters and $M$ cooperating receivers and is characterized by the inequalities

$$
\sum_{m \in \mathcal{A}} R_{m} \leq I\left(X_{\mathcal{A}} ; Y_{\mathcal{S}} \mid X_{\mathcal{A}^{c}}\right) \quad \forall \mathcal{A} \subseteq \mathcal{S}
$$

We claim that every feasible payoff profile $\underline{R}_{S}$ that lies in the capacity region $\mathcal{C}_{\mathcal{S}}$ lies in the core. By the equivalent definition of the core, in order to prove that the chosen $\underline{R}_{S}$ lies in the core, we need to show that

$$
\sum_{m \in \mathcal{G}} R_{m} \geq v(\mathcal{G}) \quad \forall \mathcal{G} \subseteq \mathcal{S}
$$

This can be shown as follows. Since $\left(R_{m}\right)_{m \in \mathcal{S}}$ is a feasible payoff profile, i.e., $\sum_{m \in \mathcal{S}} R_{m}=v(\mathcal{S})$, we have

$$
\sum_{m \in \mathcal{S}} R_{m}=\sum_{m \in \mathcal{G}} R_{m}+\sum_{m \in \mathcal{G}^{c}} R_{m}=I\left(X_{\mathcal{S}} ; Y_{\mathcal{S}}\right)
$$

We rewrite this as

$$
\begin{aligned}
\sum_{m \in \mathcal{G}} R_{m} & =I\left(X_{\mathcal{S}} ; Y_{\mathcal{S}}\right)-\sum_{m \in \mathcal{G}^{c}} R_{m} \\
& \geq I\left(X_{\mathcal{G}}, X_{\mathcal{G}^{c}} ; Y_{\mathcal{S}}\right)-I\left(X_{\mathcal{G}^{c}} ; Y_{\mathcal{S}} \mid X_{\mathcal{G}}\right) \\
& =I\left(X_{\mathcal{G}} ; Y_{\mathcal{G}}, Y_{\mathcal{G}^{c}}\right) \\
& =I\left(X_{\mathcal{G}} ; Y_{\mathcal{G}}\right)+I\left(X_{\mathcal{G}} ; Y_{\mathcal{G}^{c}} \mid Y_{\mathcal{G}}\right) \\
& \geq I\left(X_{\mathcal{G}} ; Y_{\mathcal{G}}\right)
\end{aligned}
$$

where the inequality in (13) follows from (9); (14) results from applying the chain rule for mutual information in (13), and finally (16) follows from non-negativity of mutual information. Thus, we have

$$
\sum_{m \in \mathcal{G}} R_{m} \geq I\left(X_{\mathcal{G}} ; Y_{\mathcal{G}}\right)=v(\mathcal{G})
$$

The above inequality implies that every point on the dominant face (sum-rate maximizing face of the capacity polytope $\mathcal{C}_{\mathcal{S}}$ ) of the SIMO-MAC capacity region $\mathcal{C}_{\mathcal{S}}$ corresponds to a feasible rate payoff profile that lies in the core. Thus, the core for the interference channel coalitional game is not only non-empty but is, in general, also non-unique.

\section{FAIR Allocations}

Since the interference channel coalitional game has a nonempty, non-unique core, a natural question that arises is how the value $v(\mathcal{S})$ should be apportioned between the links in a fair manner. This constitutes, in the context of coalitional games [8], a bargaining problem between all the links. Formally, a bargaining problem is a couple $(\mathcal{Q}, d)$ defined as

- a set of links $\mathcal{S}=\{1,2, \ldots, M\}$,

- the set $\mathcal{Q}$ of payoff vectors $\left(q_{m}\right)_{m \in \mathcal{S}}$ that the players can achieve through cooperation (the agreement set)

- the disagreement payoff vector $d=\left(d_{m}\right)_{m \in \mathcal{S}} \in \mathcal{Q}$ that contains the rate achieved by each of the $M$ links when it is not a part of any coalition.

A bargaining solution is a function that assigns to every bargaining problem $\langle\mathcal{Q}, d\rangle$ a unique element of $\mathcal{Q}$.

Nash Bargaining Solution Maximizing Rate Gains over Interference Channel: A solution to the bargaining problem was proposed by Nash [10] and is referred to as the Nash bargaining solution. This solution has the property that it simplifies the sequential bargaining game between links to a problem of maximizing the product of their von NeumannMorgenstern utilities [8]. We propose a Nash bargaining solution (NBS) to our bargaining problem by modeling the utility of each link as the rate gain achieved by receiver cooperation relative to the rate achieved in the non-cooperative interference channel. The resulting solution is

$$
\underline{R}_{\mathcal{S}}^{N B S}=\arg \max _{\left\{\underline{R}_{S}: R_{m}>R_{m}^{I C}\right\}} \prod_{m=1}^{M}\left(R_{m}-R_{m}^{I C}\right)
$$


where now, the agreement set $\mathcal{Q}$ is simply the SIMO-MAC capacity region $\mathcal{C}_{\mathcal{S}}$ and $R_{m}^{I C}$ is the disagreement payoff of link $m$. For the interference channel model and transmitter signaling considered, we have

$$
R_{m}^{I C}=I\left(X_{m} ; Y_{m}\right)=\log _{2}\left\{1+\frac{P_{m}\left|h_{m, m}\right|^{2}}{1+\sum_{k \neq m} P_{k}\left|h_{k, m}\right|^{2}}\right\}
$$

The Nash solution maximizes the product of the rate gains achieved by each link through cooperation. The product is maximized over the subset of the agreement set consisting of points which are strictly better than the disagreement payoffs for each player, that is, $R_{m}>R_{m}^{I C}$ for all $m$. We remark that the Nash bargaining solution [8] satisfies the properties of Pareto optimality (maximizes sum-rate) and symmetry (the solution to the bargaining problem is independent of the way the players are labeled).

Theorem 7: The Nash bargaining solution lies in the core of the interference channel coalitional game.

Proof: This is a simple consequence of the fact that the core contains all feasible payoff profiles belonging to the SIMO-MAC capacity region $\mathcal{C}_{\mathcal{S}}$ (from Theorem 7 ) and by definition 4 these feasible payoff profiles are Pareto-optimal. Since the Nash bargaining solution is always Pareto-optimal, it is a feasible payoff profile that lies in the core.

Proportional Fair Allocation: The proportional fair allocation [11] is a frequently used fairness criterion for the allocation of shared resources in communication networks. Formally, an allocation of rates $\underline{R}_{\mathcal{S}}^{P F}$ is proportionally fair (PF) if and only if for any other feasible allocation $\underline{R}_{\mathcal{S}}$, we have

$$
\sum_{m=1}^{M} \frac{R_{m}-R_{m}^{P F}}{R_{m}^{P F}} \leq 0
$$

It has been shown [11] that (20) above is equivalent to the condition $\underline{R}_{S}^{P F}=\arg \max \sum_{m=1}^{M} \log R_{m}$ which for the interference channel coalitional game simplifies as

$$
\underline{R}_{\mathcal{S}}^{P F}=\arg \left\{\max _{\left\{\underline{R}_{\mathcal{S}} \in \mathcal{C}_{\mathcal{S}}\right\}} \prod_{m=1}^{M} R_{m}\right\}
$$

Theorem 8: The proportional fair solution to the bargaining problem lies in the core of the interference channel coalitional game.

The proof follows directly from the fact that the proportional fair solution is a special case of the Nash bargaining solution where the disagreement payoff to each player is identically zero.

Thus, the Nash bargaining and proportional fair solutions in (18) and (21) respectively can be computed by limiting the search space to just the dominant face of the capacity region $\mathcal{C}_{\mathcal{S}}$. Further, limiting the search space in (21) to the constant sum-rate face implies that, for the proportional fairness strategy, the payoff vector with equal rates is the product maximizing solution provided the equal rate point lies on the dominant face of $C_{\mathcal{S}}$.
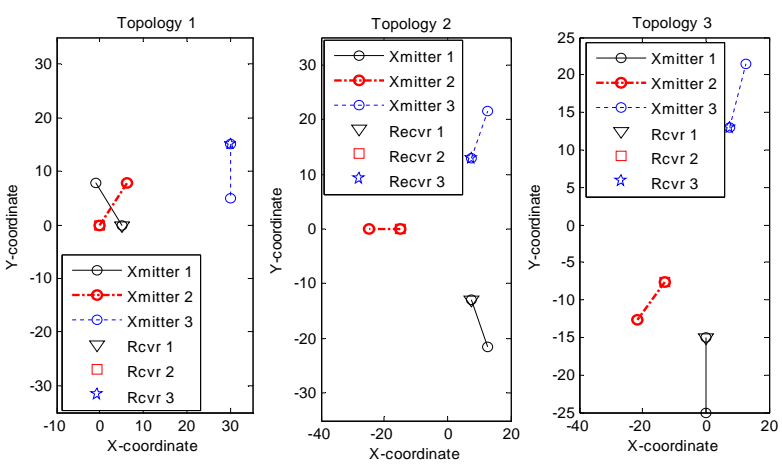

Fig. 2. Geometric plot of the three topologies

\section{ILLUSTRATION OF RESULTS}

We illustrate our results using an example of a three-link interference channel with links labeled 1,2, and 3 . We consider three network topologies as shown in Fig. 2. The channel gains for the links in each network are modeled as

$$
h_{m, k, i}=\frac{A_{m, k, i}}{d_{m, k}^{\alpha / 2}}
$$

where $\alpha$ is the path loss exponent. For the purpose of this illustration we consider a non-fading channel with $A_{m, k, i}=1$ and $\alpha=3$. Further, the transmit-receive distance $d_{m, m}$ for the $m^{\text {th }}$ link, $m=1,2,3$, is fixed at ten units while the distances $d_{m, k}, m \neq k$, are varied over the three topologies. The rate allocations are expressed in bits/channel use and all logarithms are computed to the base 2 .

In the following, for each of the three topologies, we present the rate allocations resulting from the Nash bargaining and proportional fair solutions. Note that both solutions are for a coalitional game with transferable payoff and thus the stable coalition in both cases is the grand coalition of all links, namely, $\mathcal{S}=\{1,2,3\}$. For the purpose of comparison, we also present a non-transferable payoff strategy where we apportion the value of a coalition equally between its member links; we refer to this strategy as the equal rate (ER) allocation strategy. Note that this strategy, in general, need not result in a grand coalition. In this case, the stable coalition is determined by ordering the coalitions prefered by each link based on the rate achieved and resolving the individual link preferences. For $M=3$, the total number of coalitions, given by the corresponding Bell number, is 5 .

\section{Topology 1}

Table I shows the payoff vectors (rate allocations) for the strategies of Nash bargaining and proportional fairness. These two strategies assume transferable payoff, and hence, the grand coalition is the stable coalition for both strategies. Observe that the solution for the proportional fair strategy is not the equal rate point since this point does not lie on $C_{\mathcal{S}}$. In the same table, we also enumerate the payoffs achieved by the links for the different coalition structures under the equal rate strategy where the payoffs for a coalition $\mathcal{G}$ result from distributing the 


\begin{tabular}{|l|c|c|c|c|}
\hline Coalition Structure & $R_{1}$ & $R_{2}$ & $R_{3}$ & Sum-rate \\
\hline Transferrable Payoff Allocation Strategies (NBS and PF) \\
\hline$\{1,2,3\}_{N B S}$ & 1.4391 & 1.4346 & 1.0671 & 3.9408 \\
\hline$\{1,2,3\}_{P F}$ & 1.4372 & 1.4365 & 1.0671 & 3.9408 \\
\hline Non-Transferrable Payoff Strategy (ER) \\
\hline$\{1,2,3\}$ & 1.3136 & 1.3136 & 1.3136 & 3.9408 \\
\hline$\{1,2\},\{3\}$ & 1.4174 & 1.4174 & 0.9355 & 3.7703 \\
\hline$\{2,3\},\{1\}$ & 0.4170 & 0.2055 & 0.2055 & 0.8280 \\
\hline$\{3,1\},\{2\}$ & 0.2115 & 0.4129 & 0.2115 & 0.8359 \\
\hline$\{1\},\{2\},\{3\}$ & 0.4170 & 0.4129 & 0.9355 & 1.7654 \\
\hline Stable ER Coalition: $\{1,2\},\{3\}$ & \\
\hline
\end{tabular}

TABLE I

RATE AlLOCATION FOR THE THREE STRATEGIES FOR TOPOLOGY 1

\begin{tabular}{|l|l|l|l|l|}
\hline Coalition Structure & $R_{1}$ & $R_{2}$ & $R_{3}$ & Sum-rate \\
\hline Transferrable Payoff Allocation Strategies (NBS and PF) \\
\hline$\{1,2,3\}_{N B S}$ & 0.9988 & 0.9988 & 0.9988 & 2.9964 \\
\hline$\{1,2,3\}_{P F}$ & 0.9988 & 0.9988 & 0.9988 & 2.9964 \\
\hline Non-Transferrable Payoff Strategy (ER) \\
\hline$\{1,2,3\}$ & 0.9988 & 0.9988 & 0.9988 & 2.9964 \\
\hline$\{1,2\},\{3\}$ & 0.9671 & 0.9671 & 0.9673 & 2.9015 \\
\hline$\{2,3\},\{1\}$ & 0.9673 & 0.9671 & 0.9671 & 2.9015 \\
\hline$\{3,1\},\{2\}$ & 0.9671 & 0.9673 & 0.9671 & 2.9015 \\
\hline$\{1\},\{2\},\{3\}$ & 0.9673 & 0.9673 & 0.9673 & 2.9019 \\
\hline Stable ER Coalition: $\{1,2,3\}$ \\
\hline
\end{tabular}

TABLE II

RATE ALLOCATION FOR THE THREE STRATEGIES FOR TOPOLOGY 2

value $v(\mathcal{G})$ equally between the links in $\mathcal{G}$. With knowledge of the payoffs for the different coalitions, each link chooses that coalition where it achieves the largest rate. We see from Table I that links 1 and 2 prefer the coalition structure $\{1,2\},\{3\}$ while 3 prefers the grand coalition. Since there is no rate incentive for 1 and 2 to break away from their coalition and form the grand coalition with 3 , the resulting stable coalition (core) is $\{1,2\},\{3\}$. Thus despite the grand coalition maximizing the sum-rate, it is not the stable coalition; this result is a also a consequence of the fact that the equal rate vector does not lie on the dominant sum-rate maximizing face of the threelink capacity region $C_{\mathcal{S}}$. This topology demonstrates clearly how optimal spectrum utilization need not be achieved in the absence of transferable payoff in an interference channel.

Topologies $2 \& 3$

For topology 2 with a symmetric placement of links, as shown in II and as expected, the NBS, PF, and ER strategies allocate identical rates to all the links. In topology 3 , links 1 and 2 are brought closer together compared to their positions in topology 2, while link 3 is in the same position. As shown in Table III, all three strategies result in the grand coalition and achieve the same sum-rate. Further, the ER and PF strategies are identical as a direct consequence of the fact that the equal rate point lies on the dominant face of $C_{\mathcal{S}}$. The NBS, on the other hand, allocates a greater rate to link 3 relative to the equal rate allocated to 1 and 2. This skew in rates is because the receivers of links 1 and 2 now experience greater mutual interference as compared to topology 2 .

\section{CONCLUding REMARKS}

We analyzed the formation of stable rate-maximizing coalitions between cooperating receivers in a Gaussian interference

\begin{tabular}{|l|c|c|c|c|}
\hline Coalition Structure & $R_{1}$ & $R_{2}$ & $R_{3}$ & Sum-rate \\
\hline Transferrable Payoff Allocation Strategies (NBS and PF) \\
\hline$\{1,2,3\}_{N B S}$ & 0.9868 & 0.9868 & 1.0246 & 2.9982 \\
\hline$\{1,2,3\}_{P F}$ & 0.9994 & 0.9994 & 0.9994 & 2.9982 \\
\hline Non-Transferrable Payoff Strategy (ER) \\
\hline$\{1,2,3\}$ & 0.9994 & 0.9994 & 0.9994 & 2.9982 \\
\hline$\{1,2\},\{3\}$ & 0.9774 & 0.9774 & 0.9758 & 2.9306 \\
\hline$\{2,3\},\{1\}$ & 0.9230 & 0.9209 & 0.9209 & 2.7648 \\
\hline$\{3,1\},\{2\}$ & 0.9210 & 0.9231 & 0.9210 & 2.7651 \\
\hline$\{1\},\{2\},\{3\}$ & 0.9230 & 0.9231 & 0.9758 & 2.8219 \\
\hline Stable ER Coalition: $\{1,2,3\}$ & \\
\hline \multicolumn{5}{|l|l|}{} \\
\hline
\end{tabular}

TABLE III

RATE ALLOCATION FOR THE THREE STRATEGIES FOR TOPOLOGY 3

channel using coalitional game theory. We showed that for such networks, under the assumption of transferable utility, the grand coalition maximizes spectrum utilization and the resulting coalitional game has a non-empty core. The Nash bargaining and proportional fair solutions proposed allow fair allocation of rates to the members of a coalition. The NBS particularly captures the advantage of receiver cooperation via the choice of the link utility modeled as the rate gained by being in a coalition relative that achieved in the interference channel. Receiver cooperation as studied here applies to practical networks with receivers connected either via a spectrum server or a backbone network; it is certainly of future interest to understand the coalitions formed and the rate gains achievable when the transmitters in the interference channel cooperate.

\section{REFERENCES}

[1] A. B. Carleial, "Interference channels," IEEE Trans. Inform. Th., vol. 24, no. 1, pp. 60-70, Jan. 1978.

[2] A. Sendonaris, E. Erkip, and B. Aazhang, "User cooperation diversity part I: System description,” IEEE Trans. Commun., vol. 51, no. 11, pp. 1927-1938, Nov. 2003

[3] J. N. Laneman, D. N. C. Tse, and G. Wornell, "Cooperative diversity in wireless networks: efficient protocols and outage behavior," IEEE Trans. Inform. Theory, vol. 50, no. 12, pp. 3062-3080, Dec. 2004.

[4] R. La and V. Anantharam, "A game-theoretic look at the Gaussian multiaccess channel," Advances in Network Information Theory, Proceedings of the March 2003 DIMACS workshop on Network Information Theory, DIMACS series in Discrete Mathematics and Theoretical Computer Science, vol. 66, pp. 37-42, Nov. 2003.

[5] C. Raman, R. D. Yates, and N. B. Mandayam, "Scheduling variable rate links via a spectrum server," in IEEE Symp. New Frontiers in Dynamic Spectrum Access Networks, Baltimore, Maryland, Nov. 2005, pp. 110118.

[6] O. Ileri, D. Samardzjia, T. Sizer, and N. B. Mandayam, "Demand responsive pricing and competitive spectrum allocation via a spectrum server," in IEEE Symp. New Frontiers in Dynamic Spectrum Access Networks, Baltimore, Maryland, Nov. 2005, pp. 194-202.

[7] E. Telatar, "Capacity of multi-antenna Gaussian channels," European Trans. Telecommunications, vol. 10, pp. 585-595, Nov. 1999.

[8] M. Osborne and A. Rubenstein, A Course in Game Theory. MIT Press, 1994.

[9] T. Sandholm, K. Larson, M. Anderson, O. Shehory, and F. Tohme, "Coalition structure generation with worst case guarantees," Artificial Intelligence, vol. 10, pp. 209-238, July 1999.

[10] J. F. Nash, "The bargaining problem," Econometrica, vol. 18, no. 2, pp. 155-162, Apr. 1950.

[11] F. Kelly, "Charging and rate control for elastic traffic," Euro. Trans. Telecommun, vol. 8, pp. 33-37, Jan. 1997. 